\title{
Les maîtres de langues et la constitution de la philologie romane en Allemagne
}

\section{Gerda Hassler}

\section{(2) OpenEdition \\ Journals}

Édition électronique

URL : https://journals.openedition.org/dhfles/1664

DOI : $10.4000 /$ dhfles. 1664

ISSN : 2221-4038

Éditeur

Société Internationale pour l'Histoire du Français Langue Étrangère ou Seconde

Édition imprimée

Date de publication : 1 juin 2005

ISSN : 0992-7654

Référence électronique

Gerda Hassler, "Les maîtres de langues et la constitution de la philologie romane en Allemagne »,

Documents pour l'histoire du français langue étrangère ou seconde [En ligne], 33/34 | 2005, mis en ligne le 01 janvier 2012, consulté le 27 mai 2021. URL : http://journals.openedition.org/dhfles/1664 ; DOI https://doi.org/10.4000/dhfles.1664

Ce document a été généré automatiquement le 27 mai 2021

(c) SIHFLES 


\title{
Les maîtres de langues et la constitution de la philologie romane en Allemagne
}

\author{
Gerda Hassler
}

1 On a souvent constaté que la philologie romane était née en Allemagne. Cette découverte fut souvent liée à Friedrich Diez (1794-1876), professeur à l'université de Bonn et érudit qui ne maniait aucune des langues romanes qu'il décrivait. Cette image un peu superficielle est peut-être la cause de la vision d'une rupture entre le monde pratique des maîtres de langues et celui de la scientificité d'une discipline nouvellement fondée. Je voudrais confirmer dès le début que cette position est tout à fait justifiable et qu'elle est même perpétuée, sous certains aspects, dans les universités allemandes et dans leur enseignement de la philologie romane. Mais je voudrais mettre l'accent sur les formes de transmission et d'interactions personnelles qui peuvent être constatées au cours de la formation de la philologie romane. Ce faisant, je pars, bien sûr, d'une préhistoire de la philologie romane qui ne fut pas inventée tout d'un coup par Friedrich Diez. Je m'intéresserai, de la même manière, aux formes d'application de la philologie romane à l'enseignement des langues. Pour ne pas devoir citer trop de noms, je me limiterai aux personnages qui étaient entrés en liaison avec l'Académie de Berlin.

2 La continuité entre l'âge classique et la linguistique positiviste a déjà été l'objet de plusieurs études en histoire des sciences du langage. Les protagonistes de la linguistique historico-comparative doivent beaucoup à des étymologistes comme Covarrubias, Vossius et Ménage et les sanskritistes du XVII ${ }^{e}$ siècle. Les grands catalogues des langues de la fin du XVIII et du début du XIX ${ }^{\mathrm{e}}$ siècle sont souvent considérés comme des travaux préparatoires qui n'attendaient qu'une méthode plus scientifique pour aboutir à une comparaison généalogique et typologique des langues. Sur un plan plus théorique, on a déjà discuté pour savoir comment la théorie sensualiste a imposé le point de vue génétique à un logicisme d'abord largement achronique (Droixhe 1978: 16). Tous ces développements qu'on a étudiés jusqu'ici 
surtout dans les grands textes, cités plus tard souvent comme précurseurs, semblent soulever les questions suivantes: Quel était le rôle des données linguistiques, de ce qu'on appellerait peut-être faits empiriques dans les différentes théories mises en relation? Le sensualisme ou empirisme, a-t-il vraiment contribué à une orientation vers les faits linguistiques dans leur réalité historique? Les collections de langues ont-elles fourni le matériau du développement de la linguistique historico-comparative ? Et les maitres de langues, n'ont-ils vraiment pas contribué à la linguistique scientifique par leurs descriptions de langues?

\section{Les maîtres plurilingues du XVII et $\mathrm{XVIIII}$ siècles}

3 Si le fait d'avoir travaillé sur plusieurs langues romanes et de les avoir comparées est une caractéristique des philologues allemands, il faut penser tout d'abord aux maîtres plurilingues du XVII et XVIII ${ }^{\mathrm{e}}$ siècles parmi lesquels quelques-uns s'intéressaient à beaucoup plus qu'à l'enseignement de langues. Mentionnons comme exemple Matthias Kramer (1640-1730) qui développait une large activité descriptive et didactique dans le domaine des langues romanes. Il a publié des travaux sur l'italien, le français, l'espagnol, le néerlandais et l'allemand. Les travaux de Kramer sur les langues romanes sont répartis en époques différentes. D'abord il se consacrait à la langue italienne sur laquelle il écrivit la Gran Grammatica (Vollständige Italiänische Grammatika 1674), un Dictionnaire (Das neue Dictionarium oder Wort.Buch in Italiänisch-Teutscher und TeutschItaliänischer Sprach 1676) et plusieurs ouvrages didactiques.

4 Au français, il consacrait son Dictionnaire royal, un Essay d'une bonne Grammaire Françoise (Nuremberg 1696), une collection de dialogues sous le titre Nouveau Parlement (Francfort 1696) et un Entretien de la Methode (Nuremberg 1696). Il enseignait à l'université de Heidelberg et probablement aussi à l'université d'Altdorf près de Nuremberg où il est mentionné comme Magister Linguarum exoticarum. Il passait presque dix ans à Regensburg où il était chargé de l'éducation du fils du délégué de Brandebourg auprès du Reichstag. C'était en 1712 qu'il fut élu membre de la société prussienne des sciences, pour ses mérites dans l'enseignement des langues. C'était son ancien élève, l'économiste Paul Jakob Marperger (1656-1730) qui l'avait proposé pour ses mérites dans l'enseignement des langues qui sont surtout dus à l'œuvre italienne de Kramer. Mais Kramer paraît avoir fortement aspiré à une place dans l'Académie. Dans une lettre à Marperger, il lui demande d'intervenir en sa faveur, il décrit ses mérites, donne les noms des personnes qui pourraient témoigner de sa réputation et justifie sa demande par sa volonté de dédier son dictionnaire français allemand au roi de Prusse.

5 La dominance de l'italien dans l'œuvre de Kramer s'explique certainement par les relations commerciales et culturelles de la ville de Nuremberg avec l'Italie. Kramer publiait aussi un livre de correspondance pour des commerçants italiens et allemands (Banco-Secretarius / oder kauffmännischer Correspondentz-Stylus; erkläret in drey-hundert schönen Handels-Briefen [...], Italiänisch und Teutsch, Nuremberg 1693). Dans les années 90 il avait terminé la série italienne de ses ouvrage et il commença à écrire une Gramatica y Sintaxe de la lengua Española-Castillana, publiée en 1711 qui fut sévèrement critiquée par son collègue de Leipzig Sotomayor, mais qui doit avoir eu une certaine influence en Espagne, peut-être même sur la Grammaire de l'Académie espagnole de 1771 (cf. Sánchez-Rigueira 1982, 260 ; Neumann-Holzschuh 1991, 267 ; Völker 2001, 175). C'est une grammaire qui consiste en 3 volumes, dont le premier est dédié à la phonétique et 
à la morphologie, le deuxième à la syntaxe et le troisième est un dictionnaire phraséologique. Il est remarquable que Kramer ait écrit sa grammaire espagnole en latin, prétendant vouloir atteindre un public plus large, mais certainement aussi parce que ses connaissances pratiques de la langue espagnole n'étaient pas suffisantes.

6 Sa grammaire française est une grammaire des parties du discours, classées selon le modèle latin traditionnel et incluant un livre sur la déclinaison, mais pas de livre sur les articles. Pourtant, il voit bien le problème de la transposition linéaire des catégories grammaticales du latin au français, et il donne un supplément sur la formation des cas par des prépositions qui servent de signes des cas :

III. Ein Supplementum oder accuratere Lehre von der Formatione Casuum, vermittels der Præpositionen de, à \&c. als Signis derselben / it. Von der Manier ein französisch Nomen oder Pronomen nach gestalt der Sachen ohne oder mit den Articulis le, la, les c. zu decliniren. (Essay d'une bonne Grammaire Françoise, Nuremberg 1696, 14. Seite des unpaginierten Vorberichts)

7 De la même manière, il ajoute des remarques sur l'usage des articles, domaine dans lequel, selon lui, la majorité des maitres de langues se seraient trompés. Il ne discute pas la nature de l'article, mais il donne des règles de son usage pour faciliter l'apprentissage de la langue :

IV. Von dem rechten Gebrauch und Nicht-Gebrauch gedachter Articulen / worinnen bishero die meisten Sprachmeistere geirret / und beydes / ihnen selbst das Lehren / und ihren Discipeln das Lernen und Zunehmen sauer gemacht haben . (Essay d'une bonne Grammaire Françoise, Nuremberg 1696, 14. Seite des unpaginierten Vorberichts)

8 Kramer se rend compte des problèmes de la description phonétique française et il promet une Grammaire Royale qu'il ne terminera jamais. Celle-ci contiendrait « un supplément sur la prononciation correcte et pure du français » qu'il ne savait pas encore donner. La représentation des sons français par des lettres allemandes se heurtait à des difficultés énormes. Il ne distinguait pas, par exemple, entre des consonnes sonores et sourdes, transcrivant vierge par viersche et confondant le $g$ sonore qui n'existe pas en allemand avec la consonne sourde. De plus, Kramer n'expliquait que les sons spécialement décrits par une transcription et laissait les autres dans leur orthographe française. Cela ne faisait pas voir la véritable prononciation des mots.

\begin{tabular}{|c|c|c|c|c|}
\hline graphème & $\begin{array}{l}\text { «correctement» transcrit } \\
\text { dans l'explication }\end{array}$ & lexème & $\begin{array}{l}\text { sans transcription correcte quand il } \\
\text { ne s'agit pas du son en question }\end{array}$ & lexème \\
\hline $\mathrm{ch}$ & sch (scheval) & cheval & chanté & chanter \\
\hline ce & se (seleste) & ce & recevé & recevez \\
\hline $\mathrm{Ge}$ & sche (scheneral) & ge & lange & l'ange \\
\hline ou & $u$ & ou & toute & toute \\
\hline
\end{tabular}

9 On voit bien que Kramer est loin de l'élaboration d'une méthodologie comparative ou descriptive et son activité se limitait aux manuels de langues. Il se rapproche pourtant des idées de la linguistique romane dans ses idées sur les dialectes et les langues-mères qu'il développait surtout dans ses dictionnaires. Dans ceux-ci il abandonne la 
disposition alphabétique des mots qui serait suffisante pour un dictionnaire destiné à la consultation, mais il voulait créer un dictionnaire de lecture et d'apprentissage. Pour cela, il se réclamait de l'arrangement des mots dans le premier dictionnaire de l'Académie française et il reprit la phrase fameuse «Il est agréable \& instructif de disposer le Dictionnaire par Racines... ». Pour trouver les racines, il explique les notions de 'langue originelle' ou 'langue fondamentale'. La langue originelle consistait pour lui en un certain nombre de mots créés directement par Dieu. Pour Kramer, il y avait quatre langues originelles : l'hébreu, l'allemand qu'il met en relation avec le celte, le latin et le slave. Il supposait des dérivations et des compositions des mots de ces langues qui menaient à des dialectes principaux tels que le français, l'italien, l'espagnol, l'anglais, le suédois, le danois, le polonais, le tchèque et le hongrois. Ces dialectes principaux se seraient développés au cours du temps par la corruption des quatre langues d'origine. Ils ne seraient pas encore des langues, malgré la pureté qu'ils avaient déjà acquise. A partir de ces dialectes principaux, Kramer suppose d'autres sousdialectes, issus d'une corruption continue des langues. Le français se présente pour lui comme un mélange de la langue allemande qu'il identifie au celte et au latin. Les Gaulois auraient selon lui d'abord parlé allemand, et après les romains leur auraient imposé le latin: "Ist allgemein entstanden, als die Gallier, die zuvor vermutlich Teutsch geredt, von Römern (Latinern) überzogen und untergebracht wurden ».

10 L'italien serait aussi une corruption du latin, mais plus proche de la langue d'origine. Kramer voit les causes de la corruption dans la sous-estimation du latin par les érudits, la chute de l'Empire romain et le mélange des Romains et des étrangers, finalement dans l'invasion par les Wisigoths et les peuples slaves qui auraient laissé des traces de leur vocabulaire. Tandis qu'il voit la grammaire italienne plus proche de la grecque, la grammaire et les mots de la langue espagnole seraient ceux du latin.

11 Kramer se pose la question de savoir s'il est possible d'établir un dictionnaire des racines des dialectes principaux, ce qui n'est pas possible parce qu'une langue d'origine qui ne l'est que partiellement ne dispose pas de radicaux. Pour y remédier, il se sert d'un moyen facile : il prend les mots principaux, par exemple les substantifs, les verbes et adjectifs et il les déclare comme racines, bien qu'ils ne soient que le résultat d'une corruption du latin.

12 J'ai insisté sur l'exemple de Kramer parce qu'il a publié des livres sur plusieurs langues romanes et parce que le point de vue historique ne lui était pas étranger.

13 Plusieurs maîtres de langues sont mentionnés pour leur influence sur de grands personnages. Par exemple, Johann Leonhard Frisch (1666-1743), qui était sous-recteur du lycée du Cloître gris (zum Grauen Kloster) à Berlin, enseignait les mathématiques et la physique ainsi que le grec, le latin et le français. Leibniz avait même appris le russe avec lui. Johan Nikolaus Bach (1669-1753), fils de l'organiste d'Eisenach Johann Christoph Bach et cousin de Johann Sebastian Bach, qui avait été nommé organiste de l'université à Jena, était le professeur d'italien du lecteur de français François Roux. (Schröder 1987-2001, I, 27/28)

Les maitres de langues s'éloignaient d'autant plus d'une réflexion philologique sur les langues qu'ils voulaient enseigner les langues vivantes pour ceux qui n'entendaient pas le latin. Cela exigeait le renoncement aux catégories grammaticales connues ainsi que l'exclusion de l'étymologie. Le lecteur public et maître de la langue française à l'université de Halle, David Étienne Choffin est auteur d'une série de publications, entre autres d'une Nouvelle grammaire francaise, reduite en tables, à l'usage des dames et des autres 
personnes qui ne savent pas de latin (Neue französische Grammatik, auf eine besondere Art eingerichtet und in Tabellen gebracht, zum Besten des Frauenzimmers und anderer Personen, die das Latein nicht verstehen; auf das Kürzeste, Deutlichste und Begreiflichste abgefasst, zu ungemeinem Nutzen sowohl derjenigen, welche die französische Sprache lehren, als derer, welche dieselbe lernen, vornehmlich aber Vorteil der französischen Gouvernantinnen und aller der im Französischen öffentlich Unterricht geben, 1. Auflage Berlin 1747).

Le but pratique de l'enseignement du français pour les dames empêchait la réflexion sur la provenance des formes et des sons de cette langue. Comme Choffin l'avait expliqué, dans l'enseignement pour des dames une didactique spéciale du vocabulaire était nécessaire : on ne pouvait pas s'appuyer sur la connaissance de l'étymon latin et il fallait aussi faire attention au choix des textes et expliquer la grammaire par la pratique.

16 La voie inductive de l'apprentissage de la langue française était favorisée par Jean Charles Thiébaud de la Veaux (1749-1794), maître de français à Bales qui fut appelé, juste après de fâcheuses aventures en 1779, à Berlin par Frédéric II. Il y travaillait jusqu'à 1786, et après la mort de Frédéric il se rendit à Stuttgart pour enseigner à la Hohe Karlsschule. Ensuite il s'engagea comme jacobin en Alsace où il restait jusqu'à sa mort. Il a donné une appréciation critique des ouvrages pour l'enseignement du français en Allemagne, Le maitre de langue ou Remarques instructives sur quelques ouvrages français écrits en Allemagne (Berlin 1783) qui parut de façon anonyme. Les vrais principes de la langue française oder Neue französische Grammatik für die Deutschen von einer Gesellschaft Gelehrter beider Nationen, ouvrage anonyme aussi (Berlin 1785), fait allusion à un titre bien connu en France. De plus, il publiait plusieurs livres élémentaires (Methodischer Unterricht in der französischen Sprache, 4 vol. , Berlin 1790 ; Premier livre élémentaire 1787; Cours théoretique et pratique de langue et de litterature Ouvrage entrepris par ordre du Roi, 3 vols.). De la Veaux distingue les voies de l'apprentissage des langues modernes de celles qui se prêtent à l'apprentissage d'une langue morte. Pour le français, il ne reconnaît que la routine et les règles, et il recommande pour le début la voie inductive qui commence par la routine qui est préférée par la nature dans l'apprentissage de la langue maternelle.

17 Les maîtres du français avaient des modèles différents dans la grammaire et la lexicographie françaises et ils se laissaient inspirer par des auteurs différents tels que Lancelot, Vaugelas, Girard et Pluche. La portée théorique de leurs réflexions n'était certainement pas grande, mais il y eut quand même des idées remarquables qui ont pu ouvrir la voie à la philologie romane. Bien que la méthode de Kramer ne reprenne que la discussion sur l'étymologie, sur l'harmonie des langues et les réflexions sur l'origine des langues, elle n'est pas sans méthode propre. Il donne pour chaque mot l'étymologie, la rection, la signification primaire et la signification figurée, des synonymes et des exemples d'usage. Mais il y a quelques traits qui éloignent les maîtres de plusieurs langues de la philologie romane :

18 Ils s'intéressaient surtout à la description synchronique des langues et les remarques historiques ne concernaient que l'étymologie.

19 Ils n'avaient aucune idée de l'histoire des langues et des peuples, ce qui les menait à mélanger l'idée de substrat et de superstrat dans le cas du rôle des langues germaniques pour le français.

20 Kramer n'était pas le seul maître de langues à avoir des intérêts scientifiques et à aspirer à une place à l'Académie. Mais la professionnalisation du travail linguistique 
n'était pas encore si avancée que leur activité en tant que maîtres de langues ait pu être très appréciée. Un exemple est significatif de leur situation. Un maitre de langue et comédien à la cour du comte de Bentheim-Steinfurt qui s'appelait Thiemann suivait l'ordre du comte, en 1758, de suivre des cours au gymnase Arnoldinum de Burgsteinfurt. Quand un étudiant noble, le Freiherr von Ompteda, le vit dans la classe, il refusa d'être assis côte à côte avec un comédien. Ce fut, bien sûr, le comédien et maître de langues qui dut quitter la salle.

\section{La comparaison des langues romanes dans les collections des langues : le cas du concours de l'Académie de Berlin}

21 Le tournant du XVIII ${ }^{e}$ au XIX ${ }^{e}$ siècle présente plusieurs évènements remarquables pour le développement ultérieur de la linguistique : la fondation de la Société Asiatique de Calcutta en 1783, si importante pour la reconnaissance du rôle du sanskrit, les grandes collections de langues, parmi lesquelles on mentionne souvent Pallas (1786-1789), Hervás y Panduro (1800-1805) et Adelung/Vater (1806-1817) :

De plus, la pensée des fondateurs de la linguistique historico-comparative, Rask, Bopp et Grimm, commençait à se former. Il est naturel qu'on ait surtout étudié les relations entre ces évènements sans s'occuper des maîtres mineurs qu'étaient les maîtres de langues.

23 En ce qui concerne les collections de langues, il me semble important d'élargir le point de vue et de procéder à une véritable étude sérielle. On peut même constater, dans la deuxième moitié du XVIIIe siècle, une certaine manie des collections. Dans les grandes villes d'Europe et de l'Amérique du Nord, on exposait des objets qui apportaient un témoignage sur des peuples exotiques, leurs vies et leurs cultures. Comme il était courant de considérer le génie des langues en relation avec le génie des peuples qui les parlent, il semble justifié de classer les collections de langues dans ce courant. Les collections de langues de la fin du dix-huitième et du début du dix-neuvième siècle étaient beaucoup plus nombreuses qu'on ne pense à partir des descriptions qu'on trouve dans l'historiographie de la linguistique. Nous ne donnerons qu'une liste qui comprend, en dehors des grands ouvrages connus, quelques collections et comparaisons de langues qui ont été élaborées en relation avec les activités de l'académie de Berlin.

Mentionnons d'abord le concours sur la comparaison des principales langues européennes, organisé d'abord en 1792 et renouvelé en 1794.

Vergleichung der Hauptsprachen Europas, lebender und todter, in Bezug auf Reichthum, Regelmäßigkeit, Kraft, Harmonie und andere Vorzüge; in welchen Beziehungen ist die eine der anderen überlegen, welche kommen der Vollkommenheit menschlicher Sprache am nächsten?

26 Avec cette question on invita à une comparaison impartiale des langues, après avoir proposé, pour 1784, la fameuse question des causes de l'universalité du français. Il n'y eut que trois réponses, dont le mémoire d'un prêtre berlinois, Daniel Jenisch (1796), qui remporta le prix :

Jenisch, Daniel. 1796. Philosophisch-kritische Vergleichung und Würdigung von vierzehn ältern und neuern Sprachen Europens, namentlich: der Griechischen, 
Lateinischen; Italienischen, Spanischen, Portugiesischen, Französischen ; Englischen, Deutschen, Holländischen, Dänischen, Schwedischen; Polnischen, Russischen, Litthauischen. Eine von der Königl. Preuss. Akademie der Wissenschaften gekrönte Preisschrift des Herrn D. Jenisch, Prediger in Berlin. Berlin : bei Friedrich Maurer, VIII-503 S. réussir sur une base linguistique qui n'a pas encore de méthodologie élaborée. Ce sont des critères extérieurs à la langue, notamment l'existence de la liberté de la presse et les contributions des grands philosophes et écrivains qui avaient conduit Jenisch à la constation d'une supériorité de la langue anglaise par rapport à toutes les autres langues de l'Europe. Les langues romanes suivent grâce à la grandeur de leurs nations, mais leur ordre ne serait pas encore à déterminer, sauf pour la langue portugaise que Jenisch semble totalement méconnaître :

Alles dieses zusammengenommen, welches sich bei keiner Nation jemals vereiniget hat, noch jetzt vereiniget, - verbunden mit dem Geist der Nation, welcher Schnellund Tiefgefühl, Phantasie und Urtheilskraft, Talent und Gelehrsamkeit, in einem ungewöhnlichen Grade vereiniget, - möchte ich fast behaupten, (so viel Anmassung auch eine solche Behauptung vorauszusetzen scheint) daß die Englische Sprache unter allen Europäischen Sprachen, d.h. unter allen Sprachen der Welt, den größten extensiven Reichthum hat.

Die Italienische, Spanische und Französische Sprache sind, (wenn es vielleicht auch zweifelhaft seyn dürfte, welcher von ihnen, untereinander verglichen, der Vorzug gehöre), durch die Größe und alte Berühmtheit der Nationen, deren geistiges Mittheilungs-Organ sie sind, durch die unbestreitbare Wichtigkeit einer jeden dieser Nationen für die Bildung des ganzen Europas, und durch ihre frühe und glückliche wissenschaftliche Bearbeitung - berechtiget, sogleich nach der Englischen die Stelle einzunehmen. Die Portugiesin kann sich wegen der fast immerdauernden Ruhmlosigkeit der Nation wegen der Eingeschränktheit ihres Gebrauchs, wegen des geringen Interesses dieser Nation für die Cultur des Geistes, und wegen der in allen diesen Ursachen gegründeten geringen Anzahl von Schriftstellern, keiner ihrer Lateinischen Schwestern an die Seite stellen. (Jenisch $1796: 62 / 63)$

31 Après cela, on trouve une énumération des avantages de la langue allemande(Jenisch $1796: 63)$. 

rigoureux dans son jugement. Pour l'auteur de ce manuscrit, c'est la langue grecque qui mérite d'être rétablie dans son usage et qui faciliterait la communication entre tous les peuples européens :

Diese Vollkommenheit erhielt die griechische Sprache durch die Philosophie und durch die Bemühung derer, die sich mit derselben beschäftigten. Denn ist sie es nicht, wodurch der Mensch richtig und bestimmt denken und folglich auch richtig und bestimmt reden lernt. Die Philosophen trugen auch dadurch sehr viel bey, diese Sprache zur vorzüglichsten zu machen, weil sie in ihren Schulen dieselbe gebrauchten, um ihre Schüler zu unterrichten, [...] ». (Manuscrit I-M 934. 102)

L'empirisme de ce genre de travaux est marqué par un but très clair : l'évaluation des langues à partir de critères souvent politiques et économiques. C'est dans ce sens que Jenisch avait reformulé la question de l'Académie :

Das Ideal einer vollkommenen Sprache zu entwerfen : die berühmtesten ältern und neuern Sprachen Europens diesem Ideal gemäß zu prüfen; und zu zeigen, welche dieser Sprachen sich demselben am meisten nähern ? (Jenisch 1796, III).

Il s'agit d'une comparaison évaluative dont les critères se déterminent a priori à partir de l'image idéale d'une langue parfaite. L'empirisme évaluatif de Jenisch dépasse cependant l'empirisme hypothétique du XVIII siècle sur le plan polémique. Il s'oppose à une sous-estimation de la diversité des langues, en les supposant comparables dans leurs avantages et leurs désavantages, sans se décider pour une langue qui soit la mesure de toutes les autres. Sur le plan théorique, Jenisch s'oppose aux catégories kantiennes en insistant sur l'observation des langues :

Eine einzige neue oder scharfsinnige, vielleicht nur wie zur Seite hingeworfene Bemerkung, gilt hier dem Forscher mehr, als - nach den vollständigsten Categorientabellen registrierte Alltäglichkeiten: denn die Kantischen Categorien selbst, hoffe ich, einst anderswo, für die Philosophie der Sprache fruchtbar zu benützen. (Jenisch 1796, VI).

On retrouve la même tendance évaluative dans la clefs des langues de Carlo Denina, un autre personnage lié à l'Académie de Berlin, qui suit le président de Brosses en insistant sur les différences des climats et leur influence sur les différences des langues.

En considérant la résistance qu'éprouvent certains peuples à articuler les lettres, consonnes ou voyelles, en certaines positions, ou l'habitude qu'ils ont contractée, de donner aux élémens de la parole une expression, ou une valeur différente de celle qu'ils avoient ailleurs, on devine pour ainsi dire la signification du mot qui se présente. C'est donc de là que commencent mes observations; et partant des idiomes reconnus pour pères de la plûpart des autres qu'on parle ou qu'on étudie, je fais voir comment ils sont nés, et se sont formés ; en quoi ils s'éloignent les uns des autres, et en quoi ils se ressemblent et se rapprochent réciproquement. Je prouve en même temps que la cause principale de leur différence consiste dans la variété de l'accent, effet indubitable d'une différence insensible d'organisation. J'observe ensuite que la cause secondaire de la différence des langues, je veux dire la facilité de donner aux mots une signification différente de celle qu'ils avoient dans l'idiome d'où ils sont sortis, est une suite nécessaire de la première, bien plus qu'un effet du caprice ou de la réflexion, comme on pourroit le croire. (Denina, Préface à La clef des langues [1804], 128).

Mais quittons les textes sériels qui n'occupent pas le rang des grands textes de référence. Cette dernière question de l'Académie de Berlin sur un sujet linguistique s'inscrit dans un courant européen qui mène à l'élaboration de recensements de langues. Catherine de Russie avait demandé à Peter Simon Pallas de réaliser un tel 
recensement, plus tard les notes des missionnaires jésuites réfugiés à Rome avait fourni la base d'un grand catalogue des langues du monde. Dans le plus long texte de ce catalogue, Lorenzo Hervás y Panduro traite surtout les langues amérindiennes, mais il n'est pas inutile de s'intéresser à ce qu'il a dit des langues des voisins en Europe, notamment des relations entre les langues romanes.

Le catalogue des langues de Lorenzo Hervás y Panduro qui est nettement le représentant le plus éminent des catalogues de langues, représente-t-il un nouveau type de recherches empiriques? À la suite des premières histoires de la linguistique, telles que Benfey et Thomsen, on a attribué à Hervás le rôle d'un chercheur qui préparait la base empirique de la linguistique historico-comparative. N'oublions pas que le but d'Hervás était bien différent et que les donnés linguistiques qu'on trouve dans son ouvrage font preuve d'une autre sorte d'empirisme. C'est en Italie, pays de son exil après l'expulsion des jésuites hors d'Espagne, qu'il a profité des données linguistiques rapportées du monde entier par ses confrères. Il les utilise pour élaborer son système anthropologique, qui se trouve en continuité avec l'empirisme hypothétique du XVIIIe siècle. L'interdépendance entre le génie des nations et celui de leurs langues se retrouve dans le projet d'Hervás d'établir une classification des nations sur la base des langues. Dans l'introduction à son Catalogue, il examine les différents moyens auxquels les savants ont recouru jusque-là pour fonder leurs classifications et il en vient à la conclusion que les langues sont le caractère distinctif le plus clair et le mieux approprié.

Pour la comparaison des langues, Hervás se sert de trois traits distinctifs : les mots, l'artifice grammatical et la prononciation (Hervás 1800-1805, I : 11). Tandis que les mots passent d'une langue à l'autre et varient dans leur forme et leur contenu, l'artifice grammatical reste plus constant. Une nation qui parle et pense à un moment donné d'après un certain artifice grammatical ne changera jamais celui-ci, elle sera tout au plus amenée à le perfectionner, et ce degré de perfectionnement distinguera les dialectes issus d'une même langue-mère.

El artificio particular con que en cada lengua se ordenan las palabras, no depende de la invencion humana, y menos del capricho : Él es propio de cada lengua, de la que forma el fondo. Las naciones con la civilidad y con las ciencias salen del estado de barbarie, y se hacen mas ó menos civiles y sabias : mas nunca mudan el fondo del artificio gramatical de sus respectivas lenguas. (Hervás y Panduro 1800-1805 : I, 23).

Cet artifice grammatical lui servait à rapprocher des peuples qui ne sont plus voisins, mais qui ont la même langue matrice. De cette manière, Hervás voulait découvrir les traces de l'histoire dans les langues.

Cependant, ce critère de l'artifice grammatical posait des problèmes lorsqu'il s'agissait des langues romanes. Dans la latinisation des peuples assujettis par les Romains, le passage du stade de l'emprunt de mots latins à celui de l'assimilation de l'artifice grammatical de la langue latine représente une étape importante à partir de laquelle les peuples eux-mêmes deviennent romans (Hervás 1800-1805, I : 16). Conformément à son dessein de classifier les nations sur la base des affinités et des dissemblances de leurs langues, Hervás aurait dû réunir les peuples romans dans la même famille. Pour éviter cette conclusion, il insiste sur les langues parlées par ces peuples avant la latinisation et la stabilité de ce substrat. Une observation plus approfondie du français et de l'espagnol nous montrerait, selon Hervás, que l'une de ces langues a hérité de 
beaucoup de mots du celtique et l'autre du basque qu'il prend pour la langue parlée dans toute la péninsule ibérique avant la latinisation :

El francés, que antiguamente hablaba el céltico, y el español, que antiguamente hablaba el cántabro o bascongado, actualmente hablan lenguas que son dialectos de la latina; mas quien atentamente las analice y coteje con la céltica y con la cántabra, fácilmente observará que el francés en su dialecto usa no pocos idiotismos célticos y que del mismo modo el español en su dialecto latino usa muchos idiotismos cántabros: que tanto el francés como el español, conservan muchas palabras de sus antiguos y respectivos lenguages; y que según el genio gramatical de estos, han dado terminaciones á muchas palabras latinas. (Hervás y Panduro 1800-1805 : I, 17).

41 L'utilisation des faits linguistiques dans une classification des peuples dépend nettement du but de cette classification établie avant de commencer la recherche empirique.

L'exemple de Hervás montre aussi bien que celui de Jenisch qu'on est loin des méthodes d'une linguistique historico-comparative. Les jugements sur les défauts des langues et les explications de leurs différences n'étaient pas libres de stéréotypes et ces derniers ont une stabilité remarquable.

\section{L'institutionnalisation de la philologie romane (1812-1881)}

Le développement de la philologie romane est surtout provoqué par l'intérêt qu'on prenait aux textes anciens français et provençaux. Ce courant commençait avec Raynouard, et, en Allemagne, August Wilhelm Schlegel et Friedrich Diez y répliquaient. Il est remarquable que ce domaine de recherches ait été découvert par des spécialistes d'autres disciplines. Schlegel était historien de la littérature et orientaliste à Bonn où il enseignait le sanskrit, Diez était professeur des langues moyennes et nouvelles à Bonn et il enseignait la littérature allemande jusqu'au XVI ${ }^{\mathrm{e}}$ siècle, le gotique, l'ancien et le moyen allemand et l'anglo-saxon. Le premier sujet en langues romanes qui l'intéressait était, dans l'esprit romantique, la poésie des troubadours (Diez 1826). Diez avait commencé sa carrière d'enseignement à Bonn en 1821 et, pendant les dix premières années, il n'avait pas eu beaucoup de succès. A partir de 1830, il donna des cours sur l'origine et la structure des langues romanes qui attiraient beaucoup d'étudiants.

44 Jacob Grimm, un des fondateur de la linguistique historico-comparative, s'occupait aussi de textes en ancien français et espagnol. Des questions en philologie romane n'apparaissaient pas, à cette époque, de façon indépendante. Ces questions avaient plutôt un caractère complémentaire. L'histoire des lettres modernes, commençait avec plusieurs professeurs qui se chargeaient de plus d'une langue et littérature.

La professionnalisation de la philologie romane ne se faisait donc pas à partir des langues romanes pour lesquelles on avait des maîtres bien formés et actifs dans leur travail linguistique ni à partir des collections de langues, mais à partir de l'étude des textes pour laquelle les philologues d'autres disciplines étaient plus compétents. Les premiers romanistes s'opposèrent au classicisme dans la littérature et la critiquaient dans un sens romantique. Il mettaient la réflexion historique à la place de l'appréciation traditionnelle des belles lettres. August Wilhelm Schlegel demandait :

Tout le monde se croit en état de juger les anciens temps d'après des connaissances superficielles. Le moyen le plus sûr de ne tirer aucun parti de l'histoire, c'est d'y 
porter un esprit d'hostilité. Si nous dédaignons nos ancêtres, prenons garde que la postérité ne nous le rende. (Gröber 1985, vol. 1, 103) universitaires. On créait les premières chaires d'université responsables des langues romanes: Johann Adrian à Giessen (1827), Friedrich Diez à Bonn (1830), Ludwig Gottfried Blanc à Halle (1833), Aimé Huber à Rostock (1833) et à Marburg (1836), Adelbert von Keller à Tübingen (1844), Konrad Hofmann à München (1853) et Theodor Müller à Göttingen. Dans la deuxième moitié du XIXe siècle, 26 universités allemandes avaient créé des séminaires dans lesquels on enseignait les langues romanes :

- Tübingen 1867 : Seminar für neuere Sprachen (Adelbert von Keller)

- Strasbourg 1872 : Seminar für neuere Sprachen (Eduard Boehmer)

-Wien 1872 : Seminar für französische und englische Sprache (Adolfo Mussafia)

- Heidelberg 1873 : Seminar für neuere Sprachen (Karl Bartsch)

- Freiburg im Breisgau 1874 : Seminar für neuere Sprachen (Ernst Martin), 1883 : Romanisches Seminar (Fritz Neumann)

- Halle 1875 : Seminar für Romanische Philologie (Hugo Schuchardt)

- Prag (Deutsche Universität) 1875 : Französisch-Englisches Seminar (Wendelin Foerster)

- Marburg 1876 : Romanisch-Englisches Seminar (Edmund Stengel)

- München 1876 : Seminar für neuere Sprachen und Literaturen (Konrad Hofmann, Hermann Breymann)

- Breslau 1876 : Romanisch-Englisches Seminar (Gustav Gröber)

- Berlin 1877 : Romanisch-Englisches Seminar (Adolf Tobler)

- Bonn 1878 : Seminar für romanische Sprachen (Wendelin Foerster)

- Königsberg 1878 : Romanisch-Englisches Seminar (Alfons Kissner)

- Gießen 1879 : Seminar für neuere Sprachen (Ludwig Pichler)

- Bern 1881 : Romanisches Seminar (Heinrich Morf)

- Greifswald 1981/82 : Romanisch-Englische Sozietät (Hermann Varnhagen)

- Göttingen 1882 : Romanisch-Englisches Seminar (Theodor Müller)

- Kiel 1885 : Romanisch-Englisches Seminar (Albert Stimming)

- Jena 1885 : Neusprachliches Seminar (Rudolf Thurneysen)

- Erlangen 1885 : Romanisch-Englische Sozietät (Hermann Varnhagen)

- Basel 1885 : Germanisch-Romanisches Seminar (Gustave Soldan)

- Münster 1886 : Romanisch-Englisches Seminar (Gustav Körting)

-Zürich 1887 : Romanistisch-Anglistisches Seminar (Heinrich Breitinger)

- Leipzig 1891 : Englisch-romanisches Seminar (Richard Wülker)

- Leipzig 193 : Institut für rumänische Sprache (Gustav Weigand)

- Würzburg 1893 Romanisch-Englisches Seminar (Jacob Stürzinger)

Cette institutionnalisation se faisait surtout pour former la quantité nécessaire de professeurs pour l'enseignement secondaire. Dans la plupart des séminaires, les langues romanes, et surtout le français, étaient réunies à l'anglais. On se souvient peutêtre de la fameuse phrase de Hans-Helmut Christmann selon laquelle les professeurs, une fois nommés, oubliaient le plus vite possible leur tâche principale, la formation des 
professeurs, et se consacraient à l'étude historique des langues ou bien à la lecture de textes médiévaux.

Pour les Académies, je me limiterai de nouveau à celle de Berlin où Friedrich Diez fut élu membre correspondant en 1845. Le même honneur avait été dédié en 1861 à Lorenz Diefenbach qui avait soutenu une thèse à Leipzig sur les langues romanes contemporaines avec des remarques préliminaires sur leur provenance et leurs relations de parenté (Ueber die jetzigen romanischen Schriftsprachen, die spanische, portugiesische, rhätoromanische (in der Schweiz), französische, italiänische und dakoromansiche (in mehreren Ländern des östlichen Europa's) mit Vorbemerkungen über Entstehung, Verwandtschaft usw. dieses Sprachstammes 1831). Cette thèse était livre standard sur les langues romanes jusqu'à la grammaire de Diez, parue en 1836. Mais Diefenbach ne regardait pas le développement des sons et des formes d'une manière diachronique, il confrontait plutôt des états synchroniques des langues comme l'avait fait Bopp. Loin de la méthode de Grimm, il comparait la prononciation, la relation des sons dans les langues romanes à ceux du latin et toutes les parties du discours.

Déjà en 1859, l'Université de Berlin avait proposé de nommer un professeur de langues romanes (sans toutefois créer la chaire appropriée) à la place d'un lecteur de français qui ne serait pas capable de représenter la matière d'une manière scientifique :

An unserer Universität vertritt kein Professor, wie in Bonn auf eine wissenschaftlich so ausgezeichnete Weise Prof. Dietz [sic], die romanischen Sprachen in ihrer Geschichte und Literatur - und noch hat die romanische Philologie in unserer Zeit neben der deutschen an und für sich und für die historischen Studien große Bedeutung. Es lag daher der unterzeichneten philosophischen Facultät die Frage nahe, wie weit die eingetretene Erledigung der französischen Lectur Gelegenheit und Mittel biete, die bezeichnete Lücke auszufüllen. Es erschien ihr dazu die Gründung einer Professur und zwar die Gründung einer außerordentlichen geeignet. Es verträgt sich mit dieser Ansicht sehr wohl, dass in Bonn ein so hervorragender Vertreter, wie Professor Dietz, eine ordentliche Professur bekleidet. (Archiv der Humboldt-Universität zu Berlin, Nr. 1458, Bl. 208-210, cité d'après Storost 2001, 227)

51 Un des candidats proposés, Ludwig Herrig, avait enseigné le français et l'anglais, il avait voyagé en France et il avait publié des collections de textes français pour l'usage dans les classes de français. Il me semble justifié de le considérer comme un maître de langue moderne. L'autre, Eduard Mätzner (1805-1902) avait étudié la théologie et la philologie classique avant d'apprendre le français en Suisse, où il avait travaillé comme professeur pendant deux ans. Il était recteur du lycée de filles à Berlin et on l'avait choisi pour ses travaux sur la poésie en ancien français et pour sa grammaire française qui avait une certaine importance dans l'enseignement du français (Französische Grammatik mit besonderer Berücksichtigung des Lateinischen bearbeitet, 1856). Il est impossible de voir, à partir des documents de l'archive de l'université Humboldt, pourquoi la démarche de la faculté n'a pas eu de succès. Mais la lettre du doyen laisse entrevoir qu'on voulait économiser de l'argent en embauchant seulement un professeur sur un demi poste, ce qui semblait possible avec quelqu'un qui pouvait gagner sa vie en enseignant et en remplissant ses fonctions de recteur. Le doyen avait déclaré que cela ne serait pas une solution pour une personne qui aurait besoin de loisir pour son travail savant.

Quelques années plus tard, en 1867, le pas du maître de langue vers le professeur de langue était franchi : le doyen de la faculté Moriz Haupt avait obtenu la permission de nommer un professeur titulaire d'une chaire de philologie romane à l'université de 
Berlin. Il s'adressa tout de suite à Friedrich Diez qui ne tarda pas à répondre et proposa son disciple Adolf Tobler qui devint dix ans plus tard le premier directeur du séminaire roman-anglais de l'université de Berlin. Tobler fut nommé membre de l'Académie de Berlin en 1881 et proposa par la suite des romanistes comme Gaston Paris, Ascoli et Mussafia comme membres correspondants de l'Académie. De plus, il initia une fondation Diez qui avait son siège à Berlin et qui décernait des prix pour des travaux importants. Ainsi, en 1892, 1896 et 1900 un seul savant reçut les prix de la fondation Diez: Wilhelm Meyer-Lübke pour sa grammaire des langues romanes (Lautlehre: Leipzig 1890 ; Formenlehre: Leipzig 1894; Syntax: Leipzig 1899) et sa grammaire italienne (Italienische Grammatik: Leipzig 1890) . On pourrait multiplier les noms des savants en philologie romane qui étaient liés à l'Académie de Berlin. Depuis 2001 nous disposons d'un livre de Jürgen Storost qui donne des informations sur 300 ans d'études sur les langues romanes à l'Académie qui comprend des savants de formations différentes ainsi que des maîtres de langues qui aspiraient à la reconnaissance dans le monde académique. Comme nous l'avons vu beaucoup de professeurs enseignaient une langue ou plusieurs langues avant d'être nommé dans une université.

La linguistique romane historico-comparative prend une position opposée au fonctionnalisme synchronique qui dominait dans les descriptions linguistiques des maitres de langues, tout en utilisant les mêmes catégories. Ainsi, Diez traitaient des déclinaisons et des classes des conjugaisons dans des chapitres qu'il avait consacrés aux parties du discours. Ce qui l'intéressait dans le domaine de la morphologie était la réaction des langues au changement des sons qui s'était produit à partir du latin.

Friedrich Diez introduit son livre sur la flexion par les lignes suivantes:

Les langues romanes, comme d'autres langues modernes, ont perdu une partie des anciennes formes de flexion. Il faut chercher la cause de ce phénomène dans une certaine négligence qui est naturelle au langage populaire. La prononciation de ces formes, rigoureusement soumise aux lois de la quantité, devient difficile, leur variété devient incommode ; leur son comme leur sens s'obscurcit et enfin l'esprit, tendant à la précision, cherche à combler le vide qui s'est ainsi produit dans l'organisme du langage par l'emploi de mots auxiliaires appropriés. Ces derniers sont employés soit isolément, soit comme affixes, mais d'ordinaires ils échangent leur signification individuelle contre une plus abstraite répondant à la forme grammaticale qu'ils ont charge de représenter. (Diez (1973 [1874], 1)

La langue réagit dans son ensemble aux changements survenus par la négligence de l'usage, elle se comporte comme un organisme. Et les mots qui deviennent auxiliaires subissent une perte de sens lexical concrète, ils sont grammaticalisés, comme on dirait aujourd'hui. Dans le domaine syntaxique, l'ordre des mots est présenté comme une conséquence de la perte de la flexion casuelle, « cause principale qui a privé les langues filles de la liberté presque illimitée que possède à cet égard le style classique » (Diez (1973 [1876], 413).

Meyer-Lübke veut étudier ce que la flexion latine serait devenue avec la stricte application des lois phonétiques (Lautgesetze), quelle est la relation de ces formes théoriques avec celles qui existent en réalité et quelles sont les raisons pour le décalage entre les deux. Ainsi il explique la perte des formes synthétiques de la comparaison des adjectifs par la volonté de remplacer la flexion par des périphrases compréhensibles avec des adverbes :

Das Romanische hat die lateinische Komparativbildung fast vollständig aufgegeben infolge des Bestrebens, Flexionsformen durch verständlichere Umschreibungen mittelst Adverbien zu ersetzen. So dienen denn plus und magis zur Bezeichnung 
eines höheren Grades, mitunter, z. B. im Katalanischen, auch melius und noch andere Partikeln, über die die Syntax zu handeln hat. (Meyer Lübke 1972 [1894], 83).

qu'il ne veut pas montrer l'usage de la langue, mais son développement.

Mir lag vor allem daran, Sprachentwicklung, nicht Sprachgebrauch darzustellen, daher ich mein Augenmerk vorwiegend auf die Erscheinungen lenkte, die die Veränderungen, zeigen, die uns lehre, welche Wege die Sprache einschlägt, wenn sie im Laufe der Jahrhunderte ein völlig neues Gepräge einnimmt. (Meyer-Lübke 1972 [1899], V)

La philologie romane occupe donc une position sélective à l'égard des faits linguistiques: elle ne prend en considération que les phénomènes qui montrent les changements. Sous ce point de vue, la syntaxe pose plusieurs problèmes pour lesquels on a recours à l'arrangement et aux méthodes des maîtres de langues. Le chemin de la réforme de la syntaxe proposé par Meyer-Lübke correspond à celui qu'avaient choisi les maîtres de langues. Ceux-ci avaient de plus en plus rejeté quelques catégories de la grammaire gréco-latine, Meyer-Lübke reprend cette idée, tout en affirmant qu'il ne peut pas encore y satisfaire: il ne classe donc pas le nom qui suit un verbe et la préposition pour (partir pour Paris) comme un complément d'objet, parce qu'il veut que son livre soit lisible :

Dass unsere ganze Syntax einer eingehenden Umänderung bedarf, dass sie vor allem sich von den lateinischen Kategorien loslösen muss, ist von den verschiedensten Seiten anerkannt und gefordert worden. Aber schwerer als einreissen ist neu bauen, und da stand ich nun vor der schwierigen Frage einer vollständigen Umgestaltung oder einer allmählichen. Ich habe das letztere vorgezogen aus Rücksicht auf die Benutzbarkeit meines Buches, obschon ich wohl weiss, was sich dagegen einwenden lässt; obschon es mir am allerwenigsten entgeht, dass ich, um ein Beispiel zu geben, folgerichtig auch die Verbindung eines 
Nomens mit dem Verbum durch pour, wie sie in frz. partir pour Paris vorliegt, unter die Objekte hätte einreihen sollen. (Meyer-Lübke 1972 [1899], VI) similarités avec les descriptions des maitres de langues. Leur approche n'est souvent pas vraiment historique, mais panchronique dans la mesure où ils décrivent des phénomènes et en donnent des raisons. Ainsi, c'est à partir d'exemples de langues romanes contemporaines que Meyer-Lübke explique les notions d"échelle du temps' (Zeitstufe) et de 'mode d'action' (Aktionsart) Meyer Lübke 1972 [1899], 115. Sur cette base, il discute, entre autres, l'imparfait de politesse qui désigne une action du présent ou du futur que le locuteur veut mettre en arrière plan, soulignant sa modestie et il donne un exemple de La Fontaine : «Ah ! c'est trop, lui dit-il : je voulais bien mourir, Mais c'est mourir deux fois que souffrir tes atteinte (La Font. Fables 3, 14)» Meyer Lübke 1972 [1899], 124). Il est remarquable que Meyer-Lübke commence la discussion du passé simple (qu'il appelle Perfektum 'parfait') par son renvoi à la langue littéraire. Il décrit d'abord, à la manière des maitres de langues, l'usage d'une forme verbale, avant de passer à des remarques sur le développement de cette forme et de ses fonctions (Meyer Lübke 1972 [1899], 125-136).

Chez quelques philologues romans, on trouve même des remarques sur des phénomènes du français contemporain qui ne sont pas expliqués par les maitres de langues. Par exemple, Tobler discute la formule de la question avec est-ce que et il souligne qu'elle s'emploie aussi quand le fait en question est situé dans le passé, ainsi 
que la formule de la mise en relief qu'il appelle phrase relative sans support ("beziehungsloser» Relativsatz) (Tobler 1894, 8-14). On trouve un autre exemple d'observation de la réalité linguistique chez Tobler : l'usage du passé composé du verbe modal devoir avec un infinitif pour exprimer une conclusion ou une supposition du locuteur : il a dû venir (Tobler 1894, 32-38). Il lui assigne la fonction de désigner une action supposée dans le passé dont le résultat serait présent au moment de la parole.

Dans l'étude des fonctions des formes linguistiques, les philologues des langues romanes sont très proches des maitres de langues et ils utilisent les mêmes exemples de la littérature classique.

\section{L'influence de la philologie romane sur l'enseignement des langues}

67 La philologie romane une fois institutionnalisée, a-t-elle eu une influence sur l'enseignement des langues et du français en particulier en Allemagne? La réflexion sur le lien entre les philologies modernes et l'enseignement scolaire était un but de l'Association générale allemande des philologues modernes (Allgemeiner Deutscher Neuphilologentag).

68 En Prusse, la conscience de la nécessité d'un lien entre la pratique et la théorie avait même imprégné les plans d'études de 1901 qui exigeaient le traitement scientifique de l'enseignement des langues modernes et qui prévoyaient l'égalité de celles-ci avec les autres matières du baccalauréat.

69 Très peu d'études tentent de montrer comment cette transmission du savoir linguistique se faisait dans les écoles. L'historiographie de la philologie romane s'est plutôt concentrée sur la production du savoir et non sur les maîtres mineurs qui enseignaient dans les lycées. Il y avait, entre temps, des études sur la réception des idées philologiques qui ont été faites dans l'Archiv de Herrig (depuis 1846), l'annuaire de langues et littératures romane et anglaise (Jahrbuch für romanische und englische Literatur) (depuis 1859) et, depuis 1870, dans les périodiques de didactique de langue et de pédagogie. De plus, les écrits d'écoles (Schulschriften) occupent une place importante dans la transmission du savoir de l'université à l'école. Depuis les années 20 du XIXe siècle, il était obligatoire, en Prusse et plus tard dans les autres pays allemands, de rédiger un rapport de l'école qui comprenne en plus de la chronique de l'école, des plans des leçons et des résultats des examens, un traité scientifique d'un professeur.

70 À partir de la perspective de la transmission de la théorie et pratique de la philologie romane, on peut constater quatre tendances (Kuhfuß 1976) :

- Premièrement, on exposait des résultats actuels de la recherche à des collègues ou bien à des élèves. Parmi les écrits de ce type qui ne se distinguent pas d'écrits universitaires, on trouve beaucoup de thèses.

- Dans un deuxième groupe, les résultats de la philologie romane se trouvent exposés en relation avec le public qui s'intéressait surtout à la mise en pratique et à une simplification.

- Le troisième groupe allait plus loin dans cette direction et ne regardait que l'utilisation des résultats de la recherche dans l'enseignement.

- Finalement, on trouve des écrits de programme qui vont jusqu'à la proposition de plans de cours. 
71 Ce n'est que très rarement que la place de l'école dans la transformation du savoir est déterminée, comme le faisait Ploetz qui voyait le rôle du Collège royal de Berlin dans une "pépinière de la philologie romane" (Ploetz 1851 : 14). Dans la plupart des cas le chemin de transmission est vu comme unidirectionnel de l'université à l'école. Après la réforme néohumaniste des études, il était d'abord difficile de garder la place d'une matière scientifique à côté du latin. La base de cette scientificité restait la grammaire de Diez jusqu'aux années soixante-dix. Ensuite, la réception de la phonétique et de la psychologie du langage se faisait sentir. De plus, la fréquence des traités sur des sujets en philologie romane changeait au cours du XIX siècle. Tandis que, pendant la première moitié du siècle, les écrits de programme augmentaient lentement en nombre, il y eut une explosion du chiffre de ces publications de 1874 à 1876.

Bien que la linguistique historico-comparative reste dominante, des opinions contraires s'exprimaient. Ainsi, en 1831, Wilhelm Ferdinand Heidler attaquait la nouvelle linguistique au nom de la grammaire philosophique. Mais déjà avant Diez, l'approche historique s'était imposée dans les écrits programmatiques des écoles, comme on peut voir dans l'essai de Friedrich Wilhelm Reimnitz (1829) de décrire la formation du futur dans les langues romanes sur la base de Grimm, Bopp et Buttmann et d'en donner les raisons du changement. Tandis que cet essai d'un professeur de Potsdam était plutôt confus, après la parution de la grammaire de Diez, plusieurs écrits de propagation ont été publiés. Reichhelm, auteur d'un essai d'un dictionnaire étymologique des langues romanes, était un des premiers à formuler le but pédagogique de la comparaison des langues : pour comprendre et utiliser une langue il serait indispensable de pénétrer profondément dans sa formation. La tendance à déclarer les succès de la philologie romane comme un moyen de l'enseignement d'une langue est fortement présente au milieu du XIX ${ }^{\mathrm{e}}$ siècle.

Pour démontrer cette tendance, je voudrais mentionner le traité sur l'influence de la grammaire comparée sur la méthode de l'enseignement du français du professeur de lycée W. Hornbostel (Über die Einwirkung der vergleichenden Grammatik auf die Methode des französischen Unterrichts auf Gymnasien, Programm Gymnasium Lauenburg 1857). Le principe de la comparaison est déclaré comme constitutif pour tout enseignement de langues, mais il s'exclut pour l'enseignement des langues anciennes à cause du recours nécessaire au sanskrit. C'est pourquoi la comparaison des langues romanes et du latin devient le moyen le plus important de la formation formelle des élèves. L'auteur exclut l'intérêt spécifique des élèves et déclare le principe méthodologique de la philologie romane comme principe central de l'enseignement du français. Ce n'est que très rarement que la valeur du français comme langue universelle de la communication en Europe est évoquée comme cause de son enseignement dans les lycées (Conrad 1835). Les principes didactiques, par exemple celui de la progression du plus simple au plus compliqué, ne sont que très rarement traités. Au lycée, l'enseignement du français devait prouver ses capacités de formation formelle en concurrence avec la philologie classique.

La mythologie historique qui déterminait l'idéal scientifique des philologues avait longtemps empêché la discussion des buts pratiques de l'enseignement du français. Le topique de la pureté originelle et de la valeur des institutions humaines hiérarchisait les objets scientifiques des philologies et avec eux la relation entre la philologie classique et la philologie moderne. Dans la perspective de la linguistique comparative générale, le français en tant que langue plus jeune n'était qu'une forme corrompue et 
tardive et ne pouvait atteindre qu'avec beaucoup de peine une place dans l'enseignement scientifique des lycées. La question de savoir si l'enseignement quotidien dans les lycées était vraiment empreint de la méthode historico-comparative reste pourtant ouverte. Il y a beaucoup de raisons d'en douter. Comme on peut le lire dans un plan des leçons du lycée de Potsdam de 1871, les élèves apprenaient de temps en temps quelque chose sur certains résultats éminents choisis de la grammaire romane comparée :

Mit einzelnen hervorragenden Ergebnissen der vergleichenden romanischen Grammatik werden die Schüler gelegentlich bekanntgemacht. (Lektionsplan des Gymnasiums Potsdam 1871, 15)

\section{BIBLIOGRAPHIE}

\section{Bibliographie}

ADELUNG, Johann Christoph et VATER, Johann Severin (1806-1817), Mithridates oder allgemeine Sprachenkunde mit dem Vater Unser als Sprachprobe in bey nahe fünfhundert Sprachen und Mundarten, Berlin, in der Vossischen Buchhandlung, IV Bände.

CHRIST, Herbert (1983), « Zur Geschichte des Französischunterrichts und der Französischlehrer », in Mannzmann, Anneliese (ed), Geschichte der Unterrichtsfächer I, München, Kösel, 95-117.

------(1991). « Matthias Kramer. Portrait d'un maître de langues suivi de quelques remarques concernant de futures recherches ", in Mandich, Anna Maria et Pellandra, Carla (eds.), Pour une histoire de l'enseignement du français en Italie. Actes du colloque de Parme (14-16 juin 1990), Paris, SIHFLES, 19-25.

CHRISTMANN, Hans Helmut (1983), « Romance Philology Versus English Studies in Germany in the Nineteenth Century - Selected Aspects of a Vast Subject », in Finkenstaedt, Thomas et Scholtes, Gertrude (Hrsg.), Towards a History of English Studies in Europe, Augsburg, I\&I, 283-302.

------(1985), « Romanistik und Anglistik in der deutschen Universität im 19. Jahrhundert. Ihre Herausbildung als Fächer und ihr Verhältnis zu Germanistik und klassischer Philologie », Stuttgart und Wiesbaden ( = Akademie der Wissenschaften und der Literatur, Mainz. Abhandlungen der Geistes-und Sozialwissenschafltichen Klasse, Jahrgang 1985, Nr. 1)

-----(1987), « Programmatische Texte der frühen Neuphilologie : Mager (1840), Keller (1842), Elze (1845)2, Bedeutungen und Ideen in Sprachen und Texten, Berlin, Akademie-Verlag, 51-65.

------(1991), « Romanische Philologie an den Akademien der Wissenschaften des deutschen Sprachgebietes im 19. Jahrhundert », Zeitschrift für Phonetik, Sprachwissenschaft und Kommunikationsforschung, 44/1, 4-16.

------(1994), « Linguistics and Modern Philology in Germany 1800-1840 as 'Scientific' Subject and as University Disciplines ", in Poggi, S. et Bossi, M. (Hrsg.), Romanisticism in Science, Netherlands, Kluwer Academic Publishers, 203-214. 
CONRAD, C. (1835), Die französische Sprache als Unterrichtsgegenstand für Gelehrtenschulen. Programm des Joachimsthalschen Gymnasiums Berlin, 1-34.

DENINA, Carlo (1804), La clef des langues ou observations sur l'origine et la formation des principales langues qu'on parle et qu'on écrit en Europe, Berlin, Mettra, Umlang, Quien.

DIEZ, Friedrich (1826), Die Poesie der Troubadours. Nach gedruckten und handschriftlichen Werken derselben dargestellt, Zwickau, Im Verlage der Gebrüder Schumann.

------(1973 [1874-1876]), Grammaire des langues romanes, Genève, Slatkine Reprints/ Marseille, Laffitte Reprints.

DROIXHE, Daniel (1978), La linguistique et l'appel de l'histoire (1600-1800). Rationalisme et révolutions positivistes, Genève, Librairie Droz.

GESSINGER, Joachim et RAHDEN, Wolfert von (eds.), (1989), Theorien vom Ursprung der Sprache, Berlin/New York, Walter de Gruyter.

GRIMM, Jacob (1984), « Über den Ursprung der Sprache » [1851], in Reden in der Akademie. Ausgewählt und herausgegeben von Werner Neumann und Hartmut Schmidt, Berlin, Akademie-Verlag, 64-100.

GRÖBER, Gustav (1985 [1904-1906]), Grundriss der romanischen Philologie. I. Band, Berlin/ New York, Walter de Gruyter.

GROTSCH, Klaus (1989), « Das Sanskrit und die Ursprache. Zur Rolle des Sanskrit in der Konstitutionsphase der historisch-vergleichenden Sprachwissenschaft », Gessinger et Rahden (Hrsg.), 85-121.

HASSLER, Gerda (1994), « Positionswechsel in der Behandlung der Sprachenvielfalt. Zu einigen Entwicklungen in der spanischen Sprachdiskussion von Nebrija bis Hervás », in Richard Baum et al., Lingua et Traditio. Festschrift für Hans Helmut Christmann zum 65. Geburtstag, hrsg. Tübingen, Narr 1994 : 43-56.

HEIDLER, Wilhelm Ferdinand (1831), Über philosophische Grammatik, mit besonderer Beziehung auf die französische Sprache. Programm, Frankfurt a.d. Oder.

HERVÁS Y PANDURO, Lorenzo (1787), Saggio pratico delle lingue, con prolegomeni e una racolta di orazioni dominicali in più di CCC lingue e dialetti, Cesena, Biasini.

------(1795), Escuela española de sordomudos, o arte para enseñarles a escribir y hablar el idioma español, Madrid, Imprenta Real.

-----(1979 [1800-1805]), Catálogo de las lenguas de las naciones conocidas, y numeración, división, y clases de estas, según la diversidad de sus idiomas y dialectos, Madrid, Imprenta de la Administración del Real Arbitrio de Beneficiencia 1800-1805/ Madrid, Atlas, 6 vols.

HUMBOLDT, Wilhelm von (1817), « Berichtigung und Zusätze zum ersten Abschnitte des zweyten Bandes des Mithridates über die Cantabrische oder Baskische Sprache », in Mithridates oder allgemeine Sprachenkunde mit dem Vater Unser als Sprachprobe in bey nahe fünf hundert Sprachen und Mundarten, von J. Ch. Adelung. Mit wichtigen Beyträgen zweyer großer Sprachforscher fortgesetzt von J. S, Vater, 4. Theil, Berlin, Voss'ische Buchhandlung, 275-360.

JENISCH, Daniel (1796), Philosophisch-kritische Vergleichung und Würdigung von vierzehn ältern und neuern Sprachen Europens, namentlich : der Griechischen, Lateinischen ; Italienischen, Spanischen, Portugiesischen, Französischen ; Englischen, Deutschen, Holländischen, Dänischen, Schwedischen ; Polnischen, Russischen, Litthauischen. Eine von der Königl. Preuss. Akademie der Wissenschaften gekrönte Preisschrift des Herrn D. Jenisch, Prediger in Berlin, Berlin, bei Friedrich Maurer, VIII-503 S. 
KUHFUSS, Walter (1976), « Die Rezeption der romanischen Philologie in den Programmabhandlungen der höheren Schulen im 19. Jahrhundert », in Niederehe, Hans-Josef et Harald Haarmann (eds.), In Memoriam Friedrich Diez. Akten des Kolloquiums zur Wissenschaftsgeschichte der Romanistik. Trier, 2.-4. Okt. 1975, Amsterdam, John Benjamins b.V., 327-355.

MEYER-LÜBKE, Wilhelm (1920 [1901]), Einführung in das Studium der romanischen Sprachwissenschaft, Heidelberg, Carl Winter's Universitätsbuchhandlung.

------(1972 [1894]), Grammatik der Romanischen Sprachen. II. Romanische Formenlehre, Hildesheim, New York, Georg Olms Verlag.

------(1972 [1899]), Grammatik der Romanischen Sprachen. III. Romanische Syntax, Hildesheim, New York, Georg Olms Verlag.

NEUMANN-HOLZSCHUH, Ingrid (1991), « Spanische Grammatiken in Deutschland. Ein Beitrag zur spanischen Grammatikographie des 17. und 18. Jahrhunderts », in Dahmen, Wolfgang et al. (eds.), Zur Geschichte der Grammatiken romanischer Sprachen. Romanistisches Kolloquium IV, Tübingen, Gunter Narr Verlag, 257-283

NOWAK, Elke (1996), « Considering the Status of Empirical Research in Linguistics. Approaches and Attitudes since 1800 », in Even Hovdhaugen (ed.), ... and the Word was God. Missionary Linguistics an Missionary Grammar, Münster, Nodus Publikationen, 23-44.

PALLAS Peter Simon (1786-1789), Linguarum totius orbis vocabularia comparativa, Petropoli, Schnoor.

PLOETZ, C. (1851), Quel peut être le but d'un collège français en Allemagne ? Programm Collège français royal, Berlin 1851.

RASK, Rasmus Kristian (1992), Von der Etymologie überhaupt. Eine Einleitung in die Sprachvergleichung. Hrsg. und übersetzt von Uwe Petersen, Tübingen, Gunter Narr Verlag.

REICHHELM, C. (1847), Probe eines etymologischen Wörterbuchs der romanischen Sprachen. Programm Gymnasium Stargard.

REIMNITZ, Friedrich Wilhelm (1829), Über die Bildung der Futura in de romanischen Sprachen. Programm Gymnasium Potsdam 1829.

RICKEN, Ulrich (1984), Sprache, Anthropologie, Philosophie in der französischen Aufklärung. Ein Beitrag zur Geschichte des Verhältnisses von Sprachtheorie und Weltanschauung, Berlin, Akademie-Verlag.

SÁNCHEZ-RIGUEIRA, Manuela (1982), « Un hispanista alemán del siglo XVII-XVIII y su aportación al conocimiento de la sintaxis histórica del español », in Heinz, Sieglinde et Wandruszka, Ulrich (eds.), Fakten und Theorien. Beiträge zur romanischen und allgemeinen Sprachwissenschaft. Festschrift für Helmut Stimm zum 65. Geburtstag, Tübingen, Gunter Narr Verlag, 259-266.

SCHRÖDER, Konrad (1987-2001), Biographisches und bibliographisches Lexikon der Fremdsprachenlehrer des deutschsprachigen Raumes, Spätmittelalter bis 1800, Augsburg, Universität, 7 Bände.

STOROST, Jürgen (2001), 300 Jahre romanische Sprachen und Literaturen an der Berliner Akademie der Wissenschaften, Frankfurt am Main etc, Peter Lang Verlag, 2 vols.

TOBLER, Adolf (1894), Vermischte Beiträge zur französischen Grammatik, Leipzig, Verlag von S. Hirzel.

TURGOT, Anne Robert Jacques (1961), Étymologie. 1756. Édition avec notes par Maurice Piron, Brugge, Rijksuniversiteit de Gent. 
VÖLKER, Harald (2001), « Matthias Kramer als Sprachmeister, Didaktiker und Grammatiker für die französische Sprache in Deutschland, in Dahmen, Wolfgang, Günter Holtus, Johannes Kramer, Michael Metzeltin, Wolfgang Schweickard, et Otto Winkelmann (Hrsg.), 'Gebrauchsgrammatik' und 'Gelehrte Grammatik'. Französische Sprachlehre und Grammatikographie zwischen Maas und Rhein vom 16. zum 19. Jahrhundert. Romanistisches Kolloquium XV, Tübingen, Gunter Narr Verlag, 167-250.

\section{RÉSUMÉS}

Dans cette contribution, une réponse sera donnée à la question si les maîtres de langues ont eu part à la constitution de la philologie romane en Allemagne. Je mettrai l'accent sur les formes de transmission et d'interactions personnelles qui peuvent être constatées au cours de la formation de la philologie romane. Le point de départ sera une préhistoire de la philologie romane qui n'était pas inventée tout d'un coup. Après cela, l'application de la philologie romane à l'enseignement des langues sera étudiée. Pour ne pas exagérer la citation des noms, on se limitera aux personnages qui étaient entrés en liaison avec l'Académie de Berlin.

Until now, it has been unclear whether or not language teachers have had a role in the establishment and development of Romance philology in Germany. To clarify this question, this paper will focus on the forms of transmission and the personal interaction which took place during the course of Romance philology's development. The starting point will be the prehistory of Romance philology, which was not a sudden invention. The next section of the paper will explore the application of Romance philology to language teaching. In order to avoid citing lengthy quotations and lists of names, the study is limited to persons who were in contact with the Berlin academy.

\section{INDEX}

Keywords : romance philology, institutionnalisation, Berlin academy, Germany, Diez (Friedrich)

Mots-clés : philologie romane, institutionalisation, Académie de Berlin, Allemagne, Diez

(Friedrich)

\section{AUTEUR \\ GERDA HASSLER}

Universität Potsdam 